\title{
Largest Bounding Box, Smallest Diameter, and Related Problems on Imprecise Points
}

Maarten Löffler

Marc van Kreveld

Department of Information and Computing Sciences, Utrecht University Technical Report UU-CS-2007-025

www.cs.uu.nl

ISSN: 0924-3275 


\title{
Largest Bounding Box, Smallest Diameter, and Related Problems on Imprecise Points *
}

\author{
Maarten Löffler \\ Marc van Kreveld \\ loffler@cs.uu.nl \\ marc@cs.uu.nl \\ Institute of Information and Computing Sciences \\ Utrecht University, the Netherlands
}

\begin{abstract}
We model imprecise points as regions in which one point must be located. We study computing the largest and smallest possible values of various basic geometric measures on sets of imprecise points, such as the diameter, width, closest pair, smallest enclosing circle, and smallest enclosing bounding box. We give efficient algorithms for most of these problems, and identify the hardness of others.
\end{abstract}

\section{Introduction}

Given a set of points in the plane, various measures exist that try to capture certain properties of that point set. Examples of such measures include the diameter: the largest distance between any pair of points, the closest pair: the smallest distance between any pair of points, the width: the smallest distance between two parallel lines with all points between them, the smallest circle containing all points, or the smallest axis-aligned bounding box containing all points. All these measures have been well studied and optimal algorithms to compute them are known.

When dealing with real-world data, however, locations of input points are often not known exactly. If we know for each point that it lies inside some region, but not where in that region, it becomes interesting to compute bounds on the possible values of these basic geometric measures. To make this more precise, we are given a set of regions in the plane $\mathcal{L} \subset \mathcal{P}\left(\mathbb{R}^{2}\right)$ and a measure $\mu: \mathcal{F}\left(\mathbb{R}^{2}\right) \rightarrow \mathbb{R}$ that takes a set of points and gives a real number, and we want to place one point in each region of $\mathcal{L}$ such that the resulting point set maximises or minimises $\mu$.

We study five basic measures both for maximisation and minimisation, when the imprecise points are modelled as squares or discs, possibly overlapping and of different sizes. Some of these problems have already been studied in other contexts, and efficient algorithms or hardness results are known. For most remaining problems, we present efficient algorithms here. Table 1 summarises all previous and new results. For the problem of computing the largest possible width we have not found any satisfying result, although we can prove NP-hardness when the imprecise points are modelled as line segments. For the smallest diameter of a set of discs, we have no exact algorithm but a polynomial time approximation scheme.

${ }^{*}$ This research was partially supported by the Netherlands Organisation for Scientific Research (NWO) through the project GOGO. 
Table 1: New and known results.

\begin{tabular}{|l|l|l|l|}
\hline problem & model & largest & smallest \\
\hline \hline \multirow{2}{*}{ smallest bounding box } & squares & $O(n)$ & $O(n)$ \\
& discs & $O(n)$ & $O\left(n^{2}\right)$ \\
\hline smallest enclosing circle & squares & $O(n)$ & $O(n)[7]$ \\
& discs & $O(n)$ & $O(n)$ expected \\
\hline diameter & squares & $O(n \log n)$ & $O(n \log n)$ \\
& discs & $O(n \log n)$ & $(1+\varepsilon)$-approx. in $O\left(n^{c \varepsilon^{-\frac{1}{2}}}\right)$ \\
\hline \multirow{2}{*}{ width } & squares & & $O(n \log n)[16]$ \\
& discs & & $O(n \log n)$ \\
& line segments & NP-hard & $O(n \log n)[16]$ \\
\hline closest pair & squares & NP-hard [5] & $O(n \log n)$ \\
& discs & NP-hard [5] & $O(n \log n)$ \\
\hline
\end{tabular}

\section{$1.1 \quad$ Related Work}

Data imprecision in computational geometry is often considered in stochastic or fuzzy models. However, in recent years there has been a growing interest in exact models of imprecision. Guibas et al. [6] introduce the notion of espilon geometry, a framework for robust computations on imprecise points. Abellanas et al. [1] study the tolerance of a geometric structure: the largest perturbation of the vertices such that the topology of the structure remains the same.

Some of the problems we consider here also appear in different settings. Colley et al. [4] compute the smallest area axis-aligned rectangle that intersects a set of convex polygons in $O(n \log n)$ time. Fiala et al. [5] consider the problem of finding distant representatives in a collection of subsets of a given space. In particular, they prove that maximizing the smallest distance in a set of $n$ imprecise points, modeled as circles or squares, is NP-hard. Cabello [3] gives approximation algorithms for this case. Jadhav et al. [7] consider the intersection radius of a set of objects: the smallest circle that intersects them all. Robert and Toussaint [16] develop an algorithm for computing the smallest strip that intersects a set of convex regions, while surveying several facility location problems. Such stabbing and facility location problems are related to the minimisation variants of the problems we study.

Averbakh and Bereg [2] also consider imprecise points. Where we compute the point set for which the smallest enclosing circle is worst, they compute the smallest circle that encloses the worst case point set. They study this problem in a facility location context, and they also consider weighted points and different metrics. Nagai and Tokura [12] compute the union and intersection of all possible convex hulls to obtain bounds on the solution. As imprecision regions they use discs and convex polygons, and they give an $O(n \log n)$ time algorithm. They also compute (possibly non-obtainable) lower and upper bounds for the diameter.

In [9], we also study a classical geometric problem, the convex hull, in an imprecise context. Results for the different variants of this problem range from $O(n \log n)$ time to $O\left(n^{10}\right)$ time or NP-hardness.

\section{Axis-Aligned Bounding Box}

We start with a relatively simple problem. Given a set of points $P$, the axis-aligned bounding box $(\mathrm{AABB})$ is the smallest axis-parallel rectangle that contains $P$, see Figure $1(\mathrm{a})$. In an imprecise context, we are given a set $\mathcal{L}$ of regions, and we want to place a point in each region such that 


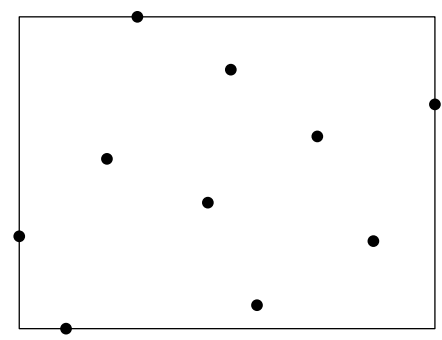

(a)

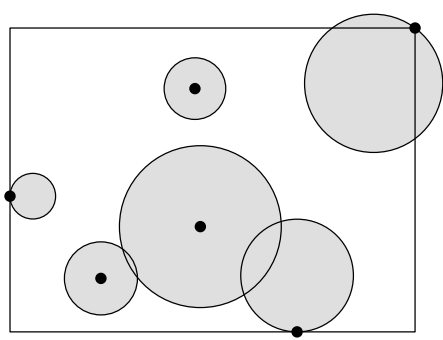

(b)

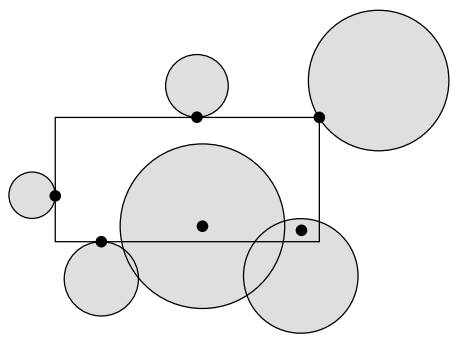

(c)

Figure 1: (a) The axis-aligned bounding box of a set of points in the plane. (b) The largest possible AABB of a set of imprecise points. (c) The smallest possible AABB of a set of imprecise points.

the bounding box of the resulting point set is as large or as small as possible, see Figures 1(b) and 1(c). We will measure the size of a rectangle by its area, but the algorithms we describe can be modified to work for the perimeter as well.

\subsection{Largest Possible AABB}

The largest possible AABB can be computed in linear time for both the square and disc model (or, in fact, any other constant complexity model). Let $\mathcal{L}^{\prime} \subset \mathcal{L}$ be the set of the four most extreme regions from $\mathcal{L}$ in the four axis-parallel directions, making sixteen elements in total. For example, the four topmost regions are the four regions for which their topmost points are the highest four.

Lemma 1 The largest possible $A A B B$ of $\mathcal{L}^{\prime}$ is equal to the largest possible $A A B B$ of $\mathcal{L}$.

Proof: Suppose this is not the case. Then there is a region $l$ in $\mathcal{L} \backslash \mathcal{L}^{\prime}$ that contributes to the $\mathrm{AABB}$ of $\mathcal{L}$, say to the top boundary. However, there are at least four regions in $\mathcal{L}$ that extend higher than $l$, of which only three can contribute to another boundary. That means we can place the point of the fourth at its topmost position, and we have a larger AABB. This contradicts the assumption.

The AABB of $\mathcal{L}^{\prime}$ can be determined by four points each lying on one of the sides of the bounding box, or by only three or two points when one or two points lie on corners. Since $\mathcal{L}$ has only constant size, we can try all possibilities and report the largest one.

Theorem 1 Given a set of $n$ squares or discs, the problem of choosing a point in each square or disc such that the axis-aligned bounding box of the resulting point set is as large as possible can be solved in $O(n)$ time.

\subsection{Smallest Possible AABB}

The smallest possible AABB is the smallest rectangle that contains at least one point of each region, so it is actually the smallest rectangle that intersects all regions. Let the left extreme line be the leftmost of the lines through the rightmost points of all regions. Similarly we define the right, top and bottom extreme lines. When the left extreme line is to the right of the right extreme line, or the top extreme line is below the bottom extreme line, there exists a zero area solution. Otherwise, they define a rectangle $R$. 


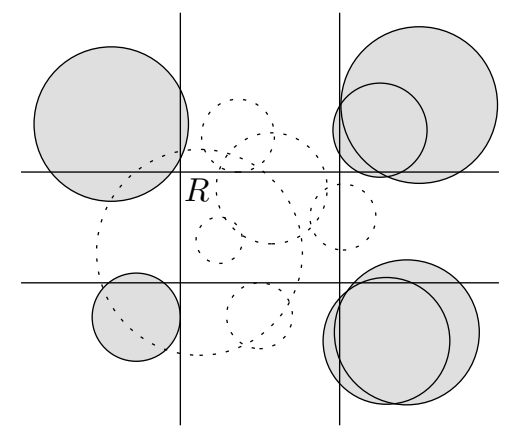

(a)

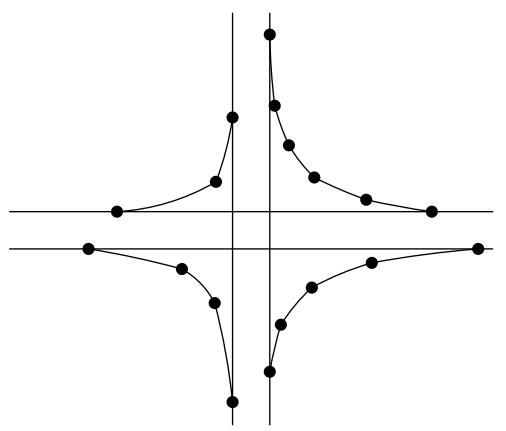

(b)

Figure 2: (a) The discs that intersect the rectangle $R$ (bounded by the extreme lines) are always accounted for. (b) The remaining discs form four chains of circular arcs.

The smallest possible AABB is the smallest rectangle that contains at least one point of each region, so it is actually the smallest rectangle that intersects all regions. Note that this rectangle must contain $R$.

\subsubsection{Squares}

When the points are modelled as squares, we know that every square must intersect $R$. Therefore, $R$ is actually the smallest AABB, and we don't need to do anything.

Theorem 2 Given a set of $n$ squares, the problem of choosing a point in each square such that the axis-aligned bounding box of the resulting point set is as small as possible can be solved in $O(n)$ time.

\subsubsection{Discs}

When the imprecise points are modelled as discs, $R$ does not necessarily intersect all discs, see Figure 2(a). Colley et al. [4] study the same problem for a set of convex polygons, and they obtain an $O(n \log n)$ time algorithm. We can use similar techniques to get a quadratic algorithm for the disc case.

We do know that $R$ needs to be contained in the smallest AABB, and therefore we do not need to consider the discs that intersect $R$ anymore. The centre points of the remaining discs lie outside the two strips between the extreme lines; this partitions them into four groups. The four corners of the smallest AABB must lie inside or behind all discs of their respective groups. We define a border between valid and invalid corner placements; these borders are convex chains of circular arcs, see Figure 2(b). We can compute these chains in $O(n \log n)$ time.

The corners of the smallest AABB must lie on or behind those four chains. This means that either the top left and bottom right corners lie on their respective chains, or the bottom left and top right corners lie on their respective chains, otherwise we could shrink the solution. We can try both these options, so suppose the top left and bottom right corners lie on the chains.

For any pair of a circle segment on the top left chain and a circle segment on the bottom right chain, we can compute the smallest rectangle with a corner on both segments in constant time. However, we need to ensure that the resulting bottom left and top right corners are on or beyond their chains as well. We do this by keeping track of the horizontal projection of the endpoints of the circle segment of the top left chain on the top right chain, and the vertical projection of 


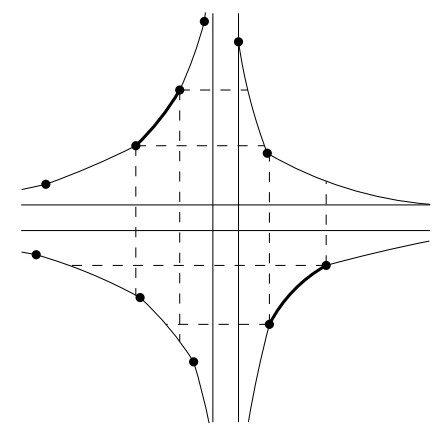

Figure 3: One arc of the top left chain and one arc of the bottom right chain are projected on the top right and bottom left chains.

these endpoints on the bottom left chain. In the same way, we keep track of the projections of the endpoints of the circle segment of the bottom right chain on the top right and bottom left chains, see Figure 3. We can clearly compute and store those projections in quadratic time.

Now, for every pair of circle segments on the top left and bottom right chains, we will compute the smallest valid rectangle with a corner on both segments. We first compute the intersections of their projections on the bottom left and top right chains. If an intersection on a chain is empty, then either this combination is impossible or it is always possible, so we don't need to consider that chain anymore. Otherwise, suppose the part of the chain in the intersection consists of $m$ circle segments. We compute the unrestricted optimal solution for the two circle segments. We can test whether it is valid in $O(m)$ time. If it is not valid, then the optimal ractangle must have a corner on this third chain. In this case, we have three chains with a point on them and we can find the optimal solution by walking over the $O(m)$ parts of the third chain and checking the smaller problems against the last chain.

Because the projections move in only one direction, the sum of the values of $m$ among all cases cannot become more than $O(n)$, so the total amount of time is $O\left(n^{2}\right)$.

Theorem 3 Given a set of $n$ discs, the problem of choosing a point in each disc such that the axis-aligned bounding box of the resulting point set is as small as possible can be solved in $O\left(n^{2}\right)$ time.

\section{Smallest Enclosing Circle}

We proceed with another relatively simple problem. Given a set of points $P$, the smallest enclosing circle (SEC) is the smallest circle that contains $P$, see Figure $4(\mathrm{a})$. When we are given a set $\mathcal{L}$ of imprecise points, we want to place a point in each region such that the SEC of the resulting point set is as large or as small as possible, see Figures 4(b) and 4(c).

\subsection{Largest Possible SEC}

\subsubsection{Squares}

The largest smallest enclosing circle of a set of squares (or constant size convex polygons) can be computed by first computing the smallest enclosing circle of the set of corners of all squares, using an existing SEC algorithm in $O(n)$ time (e.g. [10]). If the three points that determine this circle belong to different squares, we are done. Otherwise, there is one square of which multiple corners 


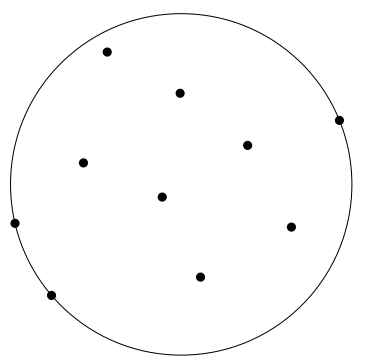

(a)

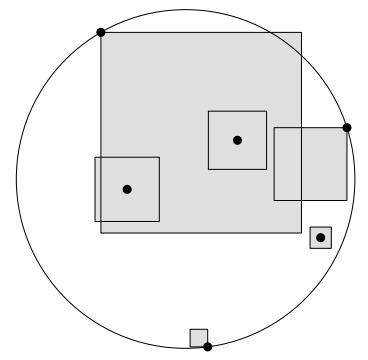

(b)

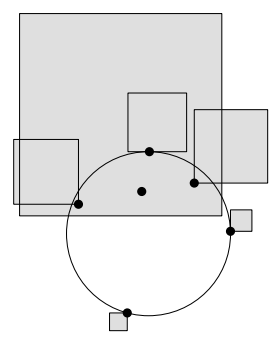

(c)

Figure 4: (a) The smallest enclosing circle of a set of points in the plane. (b) The largest SEC of a set of imprecise points. (c) The smallest SEC of a set of imprecise points.

contribute to the smallest enclosing circle, and we know that this square has to contribute to the optimal solution. So we just try all corners of this square and compute the smallest enclosing circle of this single point and all other corners of the other squares. If the result does not include this point, we have chosen the wrong one. Otherwise we know the first point of the solution, and we proceed to compute the remaining points in the same way until we have all three of them.

Theorem 4 Given a set of $n$ squares, the problem of choosing a point in each square such that the smallest enclosing circle of the resulting point set is as large as possible can be solved in $O(n)$ time.

\subsubsection{Discs}

To compute the largest possible smallest enclosing circle of a set of discs, we observe that there are only two possibilities. Either the largest SEC contains all discs, or it does not, see Figure 5. If it does, then the largest SEC is just the smallest circle containing a set of discs, which can be computed in $O(n)$ time [11]. If it does not, this means that there must be one disc among the input discs that contains all other discs. In this case, the largest SEC is determined by the point of all other discs closest to this disc, and the point on this disc furthest away from it. This case can clearly also be solved in linear time.

Theorem 5 Given a set of $n$ discs, the problem of choosing a point in each disc such that the smallest enclosing circle of the resulting point set is as large as possible can be solved in $O(n)$ time.

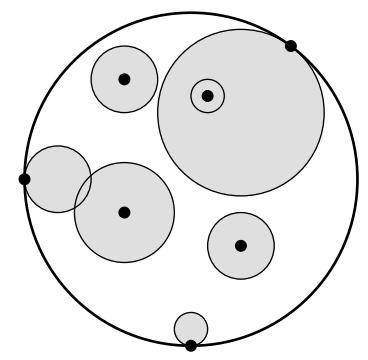

(a)

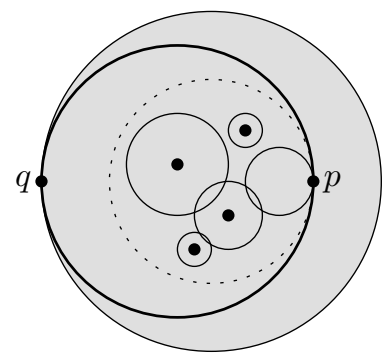

(b)

Figure 5: The LSEC (fat) of a set of discs. (a) All discs are completely within the LSEC. (b) There is one disc containing all others. 


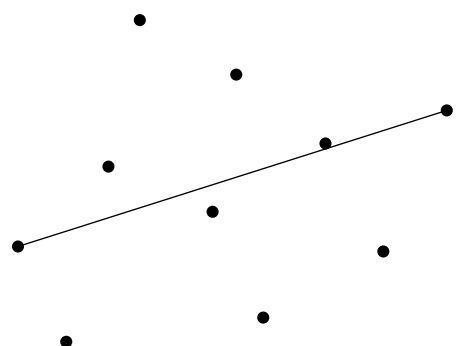

(a)

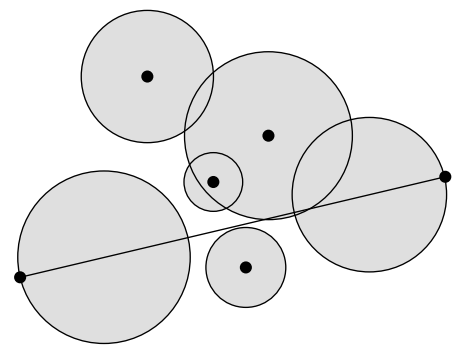

(b)

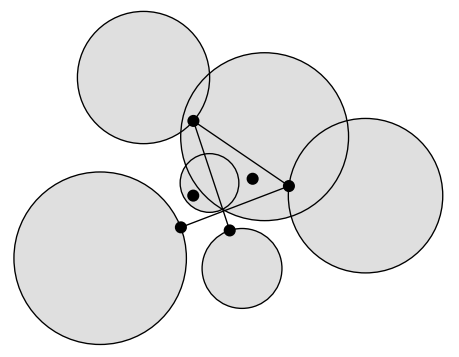

(c)

Figure 6: (a) The diameter of a set of points in the plane. (b) The largest possible diameter of a set of imprecise points. (c) The smallest possible diameter of a set of imprecise points, determined by three pairs simultaneously.

\subsection{Smallest Possible SEC}

The smallest possible SEC for a set of imprecise points is the smallest circle that intersects all regions. This is also called the intersection radius of a set of regions. When the regions are squares (or other convex polygons), it can be computed in linear time [7].

\subsubsection{Discs}

When the points are modelled as discs, we can use an adapted version of the randomised incremental construction algorithm by Welzl [18] to solve the problem.

We insert the discs in random order, keeping track of the smallest SEC of the currently inserted discs. When we insert a new disc $d$, there are two cases. If $d$ intersects the current smallest SEC, then this is still the smallest SEC of the new set of regions, because adding a region cannot make it smaller. If $d$ does not intersect the current smallest SEC, it is easy to see that the new smallest SEC must be determined by a point in $d$ and two (or one) other points. This means that we can use exactly the same recursion and time analysis as in [18], and the algorithm runs in linear expected time.

Theorem 6 Given a set of $n$ discs, the problem of choosing a point in each disc such that the smallest enclosing circle of the resulting point set is as small as possible can be solved in $O(n)$ expected time.

\section{Diameter}

Given a set of points $P$, the diameter is the largest distance between any pair of points in $P$, see Figure $6(\mathrm{a})$. When the points are imprecise, we are given a set $\mathcal{L}$ of regions, and we want to place a point in each region such that the diameter of the resulting point set is as large or as small as possible, see Figures 6(b) and 6(c).

\subsection{Largest Possible Diameter}

The largest possible diameter is formed by the pair of points among the input regions that are furthest away from each other, unless they belong to the same region. 


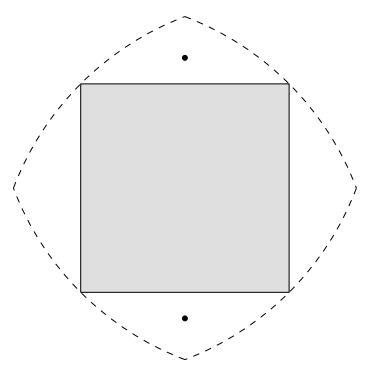

Figure 7: The largest possible diameter is formed by the two single points, even though the largest diameter among all corners is a diagonal of the square.

\subsubsection{Squares}

When the points are modelled as squares, the two points forming the largest diameter must be among the corners of the squares. This means we can just compute the diameter of the set of all corners using a conventional diameter algorithm in $O(n \log n)$ time. If the two points found belong to different squares, we are done.

Otherwise, they are diagonally opposite points of one square $s$, and there are two possibilities. Either the largest diameter is formed by one corner of $s$ and one point among the other corners. We can check all these in $O(n)$ time. The other possibility is that the largest diameter is formed by two points among the other squares, see Figure 7 .

Lemma 2 If the largest diameter of the corners of all squares is formed by two corners of one square $s$, and there are two points $p$ and $q$ in the remaining squares that are further away from each other than from $s$, then $p$ and $q$ must belong to two different squares.

Proof: Both points must lie outside $s$, because if not we could take a corner of $s$ instead and get a larger diameter. Furthermore, they must lie in two different triangle-like regions as in the figure. Now, if they would belong to the same square, this square would have to contain at least one corner of $s$, which means that it must have a corner outside $s$, and that corner with the opposite corner of $s$ would have given a larger diameter than $s$ alone, a contradiction.

Thus we can find the optimal solution by computing the diameter of the corners of all squares excluding $s$.

Theorem 7 Given a set of $n$ squares, the problem of choosing a point in each square such that the diameter of the resulting point set is as large as possible can be solved in $O(n \log n)$ time.

\subsubsection{Discs}

Compute the convex hull of the set of discs [14] in $O(n \log n)$ time, and use rotating calipers to find the diameter. We will find two points that are on some disc. If these discs are different, we are done. Otherwise, there is again one big disc that contains all others, like in the largest SEC problem, and we must find the point closest to the boundary of this disc again.

Theorem 8 Given a set of $n$ discs, the problem of choosing a point in each disc such that the diameter of the resulting point set is as large as possible can be solved in $O(n \log n)$ time. 


\subsection{Smallest Possible Diameter}

The smallest possible diameter $d$ can be determined by multiple pairs of points simultaneously, as in Figure 6(c). Moving any of the four points involved would increase the distance between at least one pair of them. In general, this could happen arbitrarily often, see Figure 11(a) where none of the points can be moved without increasing the diameter. Note that these situations are not degenerate. This makes computing the smallest possible diameter a difficult problem.

In the optimal solution, there will be some points that are exactly $d$ away from each other. In general, this will not occur at two different places, unless they depend on each other: there may be a point $p$ that has distance $d$ to two other points, and if we would move it closer to one of the other points, it would move away from the other. If this is the case, we call $p$ a bend. Note that the two other points cannot be more than $d$ away from each other, so the angle at $p$ is at most $60^{\circ}$. In the optimal solution, there can be many bends that together form a star.

For any subset $\mathcal{L}^{\prime} \subset \mathcal{L}$, let $d^{\prime}$ be the value of the smallest possible diameter of $\mathcal{L}^{\prime}$. We define the star of $\mathcal{L}^{\prime}$ to be the sequence of points that have distance exactly $d^{\prime}$ from each other in the optimal solution for this subset. This star consists of a startpoint, zero or more bends, and an endpoint, or it is cyclic with only bends. Every connection between two consecutive points in the sequence must intersect all others. Examples of stars are in Figures 6(c), 8(b) and 11(a). We call the star of $\mathcal{L}$ the optimal star.

Let $L \in \mathcal{L}$ be a region. We call $L$ an extreme region if there exists a line that has $L$ completely on one side, but no other region of $\mathcal{L}$ completely on the same side. We call a point $p \in L$ an extreme placement if such a line exists that goes through $p$. All points of the optimal star must be on extreme placements in extreme regions. Furthermore, if $p$ and $q$ are adjacent points on the optimal star, then no region is entirely on the other side than $q$ of the line through $p$ perpendicular to $\overline{p q}$.

Observation 1 Let $p$ be a point on the optimal star, and $q$ be an adjacent point on the star. Then no region is entirely on the other side of the line through p perpendicular to $\overline{p q}$.

In almost every direction, there is exactly one extreme region, except for the critical directions where a line is tangent to two regions simultaneously. If we rotate through all directions, we find the critical sequence of the regions, as introduced by Rappaport for a set of line segments [15]. When the regions are circles or squares, this sequence can be computed in $O(n \log n)$ time [13].

\subsubsection{Squares}

When the points are modelled as squares, we can solve the problem in $O(n \log n)$ time. Among the extreme squares, there are only four with infinitely many extreme placements, being the squares with the topmost bottom side, the bottommost top side, the leftmost right side and the rightmost left side. We call these squares axis-extreme. The other extreme squares can only have extreme placements at their corners. These placements form four chains: the top left chain connects all bottom right extreme placements, etc., see Figure 8(a). Note that these chains are convex. The extreme squares and chains can be computed in $O(n \log n)$ time [9].

The optimal star cannot have bends at corners of squares. If there would be a bend at a corner, it would not be possible to move the point closer to both of its neighbours, making this in fact a degenerate case and not a real bend. Therefore, the optimal star can have at most two bends, as in Figure 8(b). This implies that we can find it efficiently, as we will now show.

Lemma 3 We can find the optimal star by computing the star of every set of four extreme squares, of which two are axis-extreme, and reporting the largest among these. 


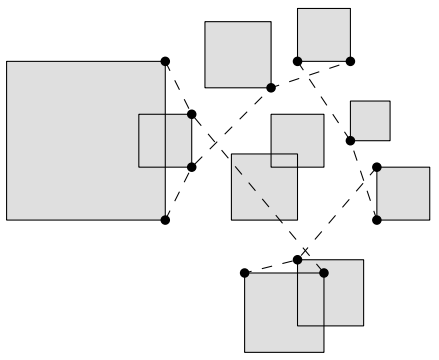

(a)

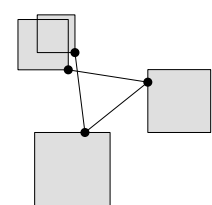

(b)

Figure 8: (a) Four extreme squares and four chains. (b) A star with two bends.

Proof: Let the reported star have value $d^{\prime}$. The optimal value $d$ must then be at least as large as $d^{\prime}$. On the other hand, it cannot be larger because then there would have been a star of length $d$ that we would have considered. Therefore, $d=d^{\prime}$.

We can compute all of these stars in $O\left(n^{2}\right)$ time. However, after precomputing the chains we can also find the optimal star in linear time, by using a careful case analysis and using the structure of the chains. For every placement of a points on an axis-extreme square, there is one vertex of a chain that is furthest away from it, and this determines the best possible diameter in this case. As the axis-extreme point moves over its edge, this furthest vertex can move only in restricted ways, which saves us a linear factor.

Assume we have computed in $O(n \log n)$ time the four axis-extreme squares and the four chains of extreme squares, as in Figure 8(a). The optimal star can be of three different types. It may be a single connection between two squares without any bends, or a star with one bend on an axisextreme square and two endpoints on the opposite chains, or a star with two bends on consecutive axis-extreme squares and two endpoints on the same opposite chain, see Figure 9. We will try all cases and all symmetric possibilities within a case, and compute the largest possible valid star in each case in linear time. The largest among these must be the optimal star.

Interval Division. In order to solve the different cases, we will need a simple structure that divides a line into intervals. Given a set of points $P$, we define a function $f(x)$ as follows. Let $L_{P}(x) \subset P$ be the set of those points in $P$ with an $x$-coordinate at most $x$. Then $f(x)$ is the furthest point in $L_{P}(x)$ from the point $(x, 0)$. We divide the $x$-axis into intervals where $f$ is constant, see Figure 10(a). There will be an unbounded interval to the left of the leftmost point where $f$ is not defined.

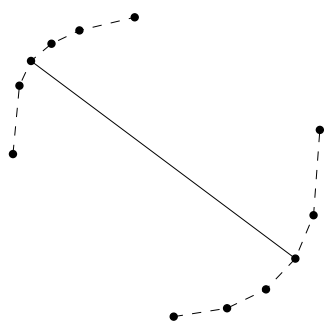

(a)

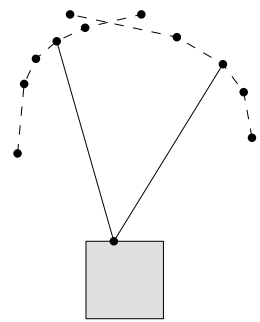

(b)

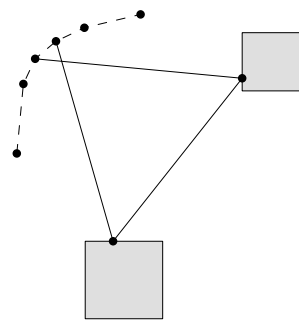

(c)

Figure 9: (a) The optimal star is a direct connection between two chain vertices. (b) The optimal star has one bend in an axis-extreme square. (c) The optimal star has two bends in consecutive axis-extreme squares. 
Lemma 4 The interval division has linear complexity and can be computed in $O(n \log n)$ time.

Proof: We can compute the interval division incrementally by sorting the points by increasing $x$-coordinate, and inserting them in that order. When we know the interval division of the $x$-axis with respect to the points $\left\{p_{1}, \ldots, p_{i}\right\}$, and we insert the next point $p_{i+1}$ with $x$-coordinate $x_{i+1}$, we observe that at most one new interval can be created and that this new interval must start at $x_{i+1}$. The new interval may (partially) overlap any number of existing intervals. However, only a linear number of intervals are created in total, therefore only a linear number are overwritten. $\square$

We define the interval division of any directed line by transforming that line onto the $x$-axis.

Case 1: no bends. When the optimal star contains no bends, it is a direct connection between two regions, see Figure 9(a). This can either be a horizontal or vertical connection between two opposite axis-extreme squares, or a diagonal connection between two vertices of opposite chains. The former case can be computed in constant time. In the latter case we need to ensure that we only consider pairs with the right slope: positive for a connection between the lower left and upper right chains, negative for a connection between the upper left and lower right chains. This can be computed by a simple variation to the conventional diameter algorithm using rotating calipers [17] in linear time.

Case 2: one bend. When the optimal star has exactly one bend, this bend is on an axisextreme square, say the bottommost square, see Figure $9(\mathrm{~b})$. The start and end points of the star must be vertices of the upper left and upper right chains. To find the largest star of this type, we must find the point $p$ on the bottommost square such that the distance to the furthest point on both chains is minimised. However, we must only consider points of the top left chain if they are to the left of $p$, and only points of the top right chain if they are to the right of $p$.

Let $l$ be the horizontal line through the top side of the bottommost axis-extreme square. We compute the interval division of the directed line $l$ from left to right with respect to the set of points that form the top left chain, and we also compute the interval division of $l$ directed from right to left with respect to the points in the top right chain. We can now balance the furthest points on both chains in linear time.

Case 3: two bends. When the optimal star has two bends, these bends must occur at consecutive extreme squares, say the bottommost and rightmost ones, see Figure $9(\mathrm{c})$. The start and end points are then vertices of the top left chain. We must now find the point $p$ on the bottom square and the point $q$ on the right square that minimise the largest among the distance from $p$ to the furthest vertex of the chain to the left of $p$, from $q$ to the furthest vertex of the chain above $q$, and from $p$ to $q$.

To find these optimal positions, we again compute the interval division of the line from left to right through the top side of the bottommost axis-extreme square with respect to the points on the top left chain, and we similarly partition the line from top to bottom through the left side of the rightmost axis-extreme square with respect to the same point set. Now we place $p$ at its local optimal position, and $q$ too. The furthest distance must occur between $p$ and $q$, otherwise the optimal star was not of this type. Now start moving the points towards each other, keeping their distances to the furthest point on the chain equal. They both move only in one direction, so we can find the optimal location in linear time again.

Placing the points. We have now computed the value $d$ of the smallest possible diameter. If needed, we can also compute a placement of the points in their regions that realises this diameter. We first compute valid placements of the four axis-extreme points, and then observe that all other points should be placed 'as far inward' as possible. 


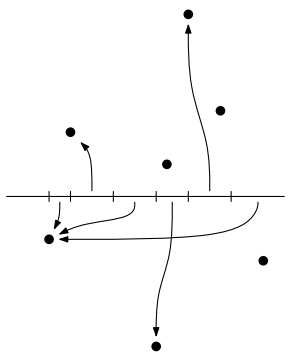

(a)

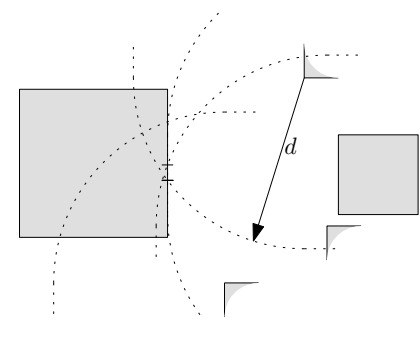

(b)

Figure 10: (a) The interval division of a set of 7 points. (b) The leftmost axis-extreme points must be placed within the dotted circles.

To compute a valid placement in an axis-extreme square, note the following. If we find a placement such that any star that includes this point has at most length $d$, then this placement is valid for a global solution of diameter $d$. This means we can check for all possible stars in which interval the point is allowed to lie if that star must be at most $d$ long, and then place the point somewhere in the intersection of all these intervals.

To determine the intervals of an axis-extreme square, say the bottommost, where a point could be placed that still allows for a solution of length $d$, we just compute for every square the interval that is at most $d$ away from that square. The intersection of these intervals gives an interval where a point can still be placed that is at most $d$ away from any other square, see Figure 10(b). After that, we must still place the axis-extreme points in such a way that they are at most $d$ away from each other. However, we already know that this is possible and since there are only four axis-extreme points we can place them in constant time. Any placement within the precomputed intervals will be fine.

For the rest of the squares, if a point is to the left of the vertical lines through the topmost and bottommost axis-extreme points, moving it to the right can only decrease the diameter, and a similar statement holds for the other extremes. Because the regions are squares, every point will end either in a corner of its region or somewhere in the middle of the whole construction.

Theorem 9 Given a set of $n$ squares, the problem of choosing a point in each square such that the diameter of the resulting point set is as small as possible can be solved in $O(n \log n)$ time.

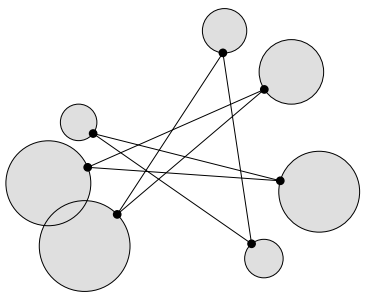

(a)

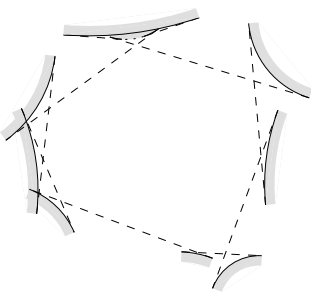

(b)

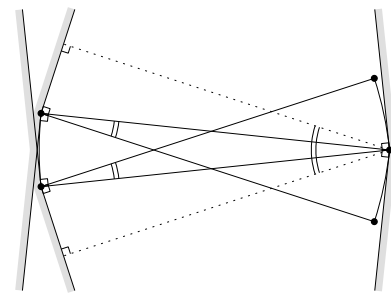

(c)

Figure 11: (a) A cyclic star that visits seven regions. (b) Circular arcs that are extreme in some direction. (c) Three consecutive angles of at most $\alpha$ give a diameter of $\cos \alpha$. 


\subsubsection{Discs}

When the points are modelled as discs, stars can have up to $n$ bends, see Figure 11(a). This leads to algebraic difficulties: even if we would know the combinatorial structure of the optimal star, computing it exactly would not be possible.

We can make some observations about the combinatorial structure. We can still define the extreme discs, that is, discs that have a tangent line with no other disc completely on the same side. These discs form a chain of arcs with this property, see Figure 11(b). Any bend in the star still needs to be on such an extreme arc. However, it is possible that all discs have extreme arcs.

Since we cannot compute the optimum efficiently, we approximate. A factor $\frac{2}{3} \sqrt{3} \approx 1.15$ approximation can be computed in linear expected time by computing the smallest smallest enclosing circle of the imprecise points. We can also compute a $(1+\varepsilon)$-approximation of the smallest diameter in $O\left(n^{c \varepsilon^{-\frac{1}{2}}}\right)$ time.

Constant factor approximation. The approximation is based on the fact that for any given point set $P$, the diameter $d$ of $P$ is at least $\sqrt{3}$ times the radius $r$ of the smallest enclosing circle of $P$. This is because of the three points that define the SEC, there must be two that are at least as far apart as if they lay on an equilateral triangle. Also, $d$ can at most be $2 r$.

Now let $\mathcal{L}$ be a set of imprecise points, let $P$ be a set of points that realises the smallest possible diameter and let $Q$ be a set of points that realises the smallest possible SEC.

Lemma 5 The diameter of $Q$ is at most $\frac{2}{3} \sqrt{3}$ larger than the diameter of $P$.

Proof: Let $d_{P}$ be the diameter of $P, d_{Q}$ the diameter of $Q, r_{P}$ the radius of the SEC of $P$ and $r_{Q}$ the radius of the SEC of $Q$. Then we have:

$$
d_{Q} \leq 2 r_{Q} \leq 2 r_{P}=\frac{2}{3} \sqrt{3}\left(\sqrt{3} r_{P}\right) \leq \frac{2}{3} \sqrt{3} d_{P}
$$

Since we can compute the smallest SEC in linear expected time, we can approximate the smallest diameter within a factor $\frac{2}{3} \sqrt{3}$ in linear expected time as well.

Approximation scheme. We can compute a $(1+\varepsilon)$-approximation in $O\left(n^{3 \pi \varepsilon^{-\frac{1}{2}}}\right)$ time. The idea of this algorithm is to consider only stars of at most $k$ bends, for $k$ chosen suitably. We compute a subset of $\mathcal{L}$ with an optimal solution which is a factor $\left(1-\frac{1}{2} \varepsilon\right)$ shorter than the real optimum; if we divide this value by $\left(1-\frac{1}{2} \varepsilon\right)$ we get a $\left(1+\frac{1}{2} \varepsilon+o\left(\varepsilon^{2}\right)\right)$-approximation, which is an $(1+\varepsilon)$-approximation if $\varepsilon \leq 1$.

Lemma 6 Suppose the optimal solution is given by a star of at least $k$ bends. Then there exists a star with only one bend that approximates it within a factor of $1-O\left(k^{-2}\right)$.

Proof: Suppose that the optimal diameter is 1 . The sum of the angles that the star makes in the bends is at most $\pi$. That means that there are three consecutive bends $a, b$ and $c$ somewhere that together make an angle of at most $\alpha=\frac{3 \pi}{k}$. Therefore the individual angles are also at most $\alpha$, see Figure 11(c).

The region of $b$ is convex and completely outside the wedge that is formed by the halfline from $b$ perpendicular to $\overline{a b}$ in the direction of $c$, and the halfline from $b$ perpendicular to $\overline{b c}$ in the direction of $a$, otherwise $b$ would not be in its optimal position. In the same way, the regions of $a$ 


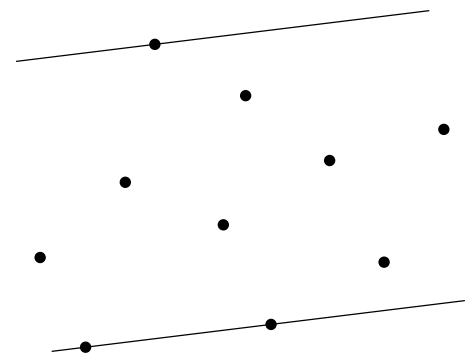

(a)

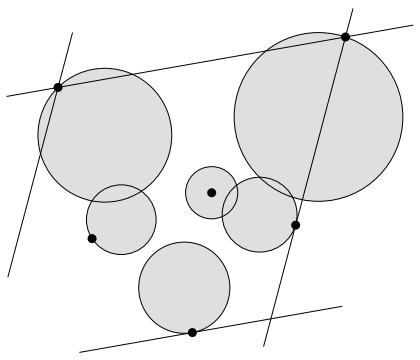

(b)

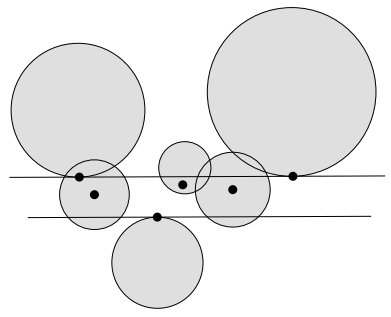

(c)

Figure 12: (a) The width of a set of points in the plane. (b) The largest possible width of a set of imprecise points, reached at two different locations simultaneously. (c) The smallest possible width of a set of imprecise points.

and $b$ are also convex and behind the wedges formed by their two neighbours on the star, unless one of them is the endpoint of the star and the wedge degenerates to a line.

This means that in the worst case the regions of $a$ and $c$ are arbitrarily close to the halflines that come nearest to $b$, and in that case the best possible diameter of $a, b$ and $c$ would be $\cos \alpha$, as denoted by the dotted lines in Figure 11(c). This is more than $1-\frac{1}{2} \alpha^{2}=1-\frac{9 \pi^{2}}{2 k^{2}}$.

To get a $\left(1-\frac{1}{2} \varepsilon\right.$ )-approximation we take $k=3 \pi \varepsilon^{-\frac{1}{2}}$ (and at least 3 ) and compute all chains of length at most $k$ in $O\left(n^{k}\right)$ time. If the optimal star has more than $k$ bends, there is a good approximation with only three bends; otherwise, we find the optimum.

Theorem 10 Given a set of $n$ discs, the problem of choosing a point in each disc such that the diameter of the resulting point set is as small as possible can be approximated within a factor $(1+\varepsilon)$ in $O\left(n^{c \varepsilon^{-\frac{1}{2}}}\right)$ time.

\section{$5 \quad$ Width}

Given a set of points $P$, the width is the smallest distance between any pair of parallel lines that contains $P$, see Figure 12(a). Examples of the imprecise case are in Figures 12(b) and 12(c).

\subsection{Largest Possible Width}

This problem seems to be hard. When the points are modelled as line segments, it is even NP-hard.

\subsubsection{Line Segments}

Computing the largest possible width of a set of imprecise points modelled as arbitrarily oriented line segments is NP-hard. We prove this by reduction from SAT. The construction is similar to the one used to prove NP-hardness of computing the largest possible convex hull of a set of line segments [8], but the nature of the width measure requires some new ideas.

Given a SAT instance, let $k$ be odd and larger than the number of clauses and variables in the instance together. We base the contruction on a regular $2 k$-gon. We place $k$ precise points distributed evenly on the vertices of the $2 k$-gon, see Figure 13(a). Let the furthest distance between two of these precise points be $d$. The imprecise points that we will place later will all be completely 


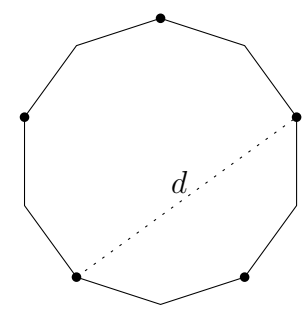

(a)

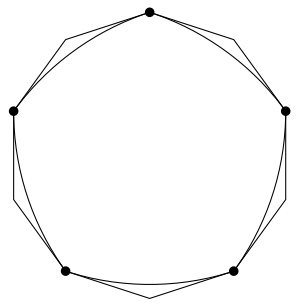

(b)

Figure 13: (a) A regular 2k-gon with width $d$. (b) A curve of constant width, formed by $k$ arcs.

within the $2 k$-gon. This ensures that the largest width can at most be $d$, because the width of the $2 k$-gon itself is $d$. We will make a construction such that a width of $d$ arises if and only if the SAT instance can be satisfied.

Now, for all $k$ precise points we draw an arc with that point as center between its two opposite points. These arcs together form a curve of constant width, see Figure 13(b). This is the smallest possible region within the $2 k$-gon that contains the $k$ precise points and has width $d$. As a consequence, a solution of width $d$ is possible if and only if a solution the imprecise points can be placed in such a way that the convex hull of the points completely contains this curve of constant width. There are $k$ parts between the $2 k$-gon and the curve of constant width, see Figure $14(\mathrm{a})$, which we will use to construct variables and clauses.

Lemma 7 A solution has width $d$ if and only if the convex hull of the placed points completely contains the curve of constant width figure (and stays within the $2 k$-gon).

Proof: If the convex hull of the points completely contains the figure of constant width, its width must be at least $d$. On the other hand, it is still completely contained in the $2 k$-gon, which also has width $d$, so its width is at most $d$. Therefore, it is $d$.

If the convex hull of the points does not completely contain the figure of constant width, there is some point $p$ in the interior of the figure that is not inside the convex hull. This means that there is a line $l$ through $p$ that does not intersect the convex hull, and therefore does not intersect the $k$-gon formed by the $k$ precise points. Because $k$ is odd, there is a line parallel to $l$ through one of the $k$ precise points that does not intersect the $2 k$-gon anywhere else, and since this point is part of the figure of constant width the distance between these two lines is less than $d$. Since the convex hull is completely between them, the width of the placed points is also less than $d . \quad \square$

For each Boolean variable $b$ in the SAT instance, we add the configuration of Figure 14(b), consisting of two points $t$ and $f$ and two sets of points $T$ and $F$, inside an empty part. We can only use one of the points $t$ and $f$, so if the convex hull of the resulting point set must contain the

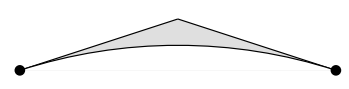

(a)

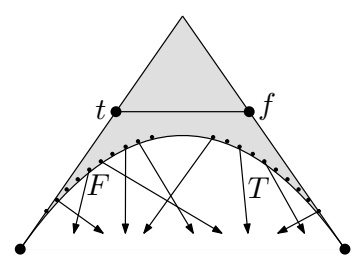

(b)

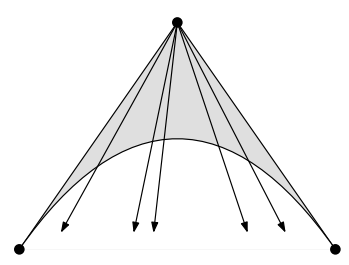

(c)

Figure 14: (a) A part between the $2 k$-gon and the curve of constant width. (b) A variable inside a part. (c) A clause inside a part. 


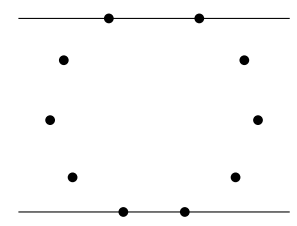

(a)

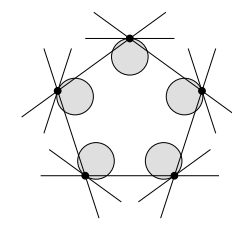

(b)

Figure 15: (a) A set of $n$ points, such that the width changes when any of them is removed. (b) Many triples of points define the width simultaneously.

circular arc we need either $t$ and all points in $T$ (representing the value true of this variable) or $f$ and all points in $F$ (representing false). For each clause we add a single point $s$ at the top of an empty part, see Figure 14(c). We include a line segment (an imprecise point) between $s$ and every variable in this clause. If the variable occurs normally, the other endpoint is in the $T$-group, and if it is negated the other endpoint is in the $F$-group. Since we can use only one endpoint of every segment, we can only place a point at $s$ if at least one of the variables of the clause is in the right state.

Theorem 11 Given a set of $n$ arbitrarily oriented line segments, the problem of choosing a point on each segment such that the width of the resulting point set is as large as possible is NP-hard. The decision version of the problem is NP-complete.

\subsubsection{Squares or Discs}

The width of a set of points has the property that there can be points that do not contribute to the width, but are needed to make the width valid, see Figure 15(a). Removing any point here would result in a smaller width. In an imprecise context, this means that the placement of all points is important, and we cannot look only at subsets of the regions. Furthermore, it can happen that many points are simultaneously involved in the optimal width, see Figure 15(b), in a similar way as the stars in the smallest diameter problem. The status of the largest width problem for imprecise points modelled as squares or discs is open.

\subsection{Smallest Possible Width}

The smallest width of a set of imprecise points can be computed efficiently. For squares (or any polygonal regions), the problem can be solved in $O(n \log n)$ time [16].

For discs, we compute the critical sequence of extreme placements again, see Figure 11(b), in $O(n \log n)$ time [13]. The smallest width must have a critical line on one side, so we can find it by using rotating calipers.

Theorem 12 Given a set of $n$ discs, the problem of choosing a point in each disc such that the width of the resulting point set is as small as possible can be solved in $O(n \log n)$ time.

\section{Closest Pair}

We conclude with another related problem. Given a set of points $P$, the closest pair is the pair of points with the smallest distance between them among all pairs of points in $P$, see Figure 16(a). In an imprecise context, we are given a set $\mathcal{L}$ of regions, and we want to place a point in each 


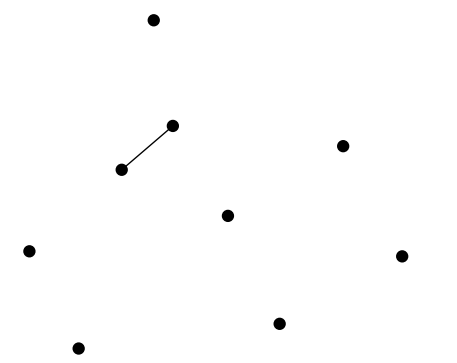

(a)

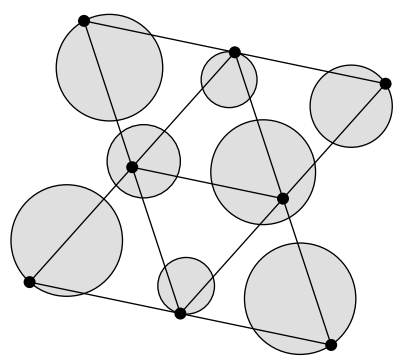

(b)

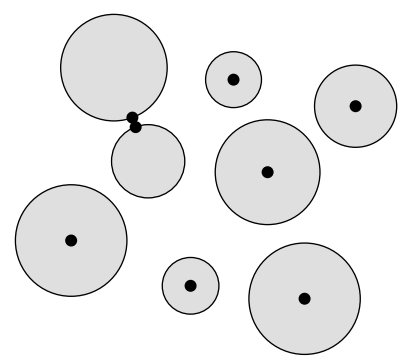

(c)

Figure 16: (a) The closest pair of a set of points in the plane. (b) The largest possible closest pair of a set of imprecise points, reached by many pairs simultaneously. (c) The smallest possible closest pair of a set of imprecise points.

region such that the closest pair of the resulting point set is as large or as small as possible, see Figures 16(b) and 16(c).

\subsection{Largest Possible Closest Pair}

Maximising the closest pair distance means that we need to place all points in such a way that their mutual distances are balanced. This is known as spreading points. Fiala et al. [5] proved this to be NP-hard, both in the square and the circle model. Cabello [3] gives a constant factor approximation algorithm.

\subsection{Smallest Possible Closest Pair}

To minimise the closest pair distance, we just need to find the two regions that are closest to each other and place their points as close together as possible. First, we check whether any two regions intersect each other in $O(n \log n)$ time by a standard sweep. If there is any intersection, the smallest possible closest pair has distance 0. Otherwise, we compute the Voronoi diagram of a set of convex objects [19] in $O(n \log n)$ time. The closest pair is determined by objects in adjacent cells, so we can find them efficiently.

Theorem 13 Given a set of $n$ squares or discs, the problem of choosing a point in each square or disc such that the closest pair of the resulting point set is as small as possible can be solved in $O(n \log n)$ time.

\section{Conclusions}

We have given a structured overview of the imprecise variants of several basic geometric measures, reusing known results from various other contexts and designing efficient algorithms for the remaining cases. Most problems can be computed efficiently, while some are NP-hard. One remaining open problem is that of computing the largest possible width for the square or disc model. Also, the 3D versions of most problems are still open. 


\section{References}

[1] M. Abellanas, F. Hurtado, and P. A. Ramos. Structural tolerance and Delaunay triangulation. Inf. Proc. Lett., 71:221-227, 1999.

[2] I. Averbakh and S. Bereg. Facility location problems with uncertainty on the plane. Discrete Optimization, 2:3-34, 2005.

[3] S. Cabello. Approximation algorithms for spreading points. Journal of Algorithms, to appear.

[4] P. Colley, H. Meijer, and D. Rappaport. Optimal nearly-similar polygon stabbers of convex polygons. In Proc. 6th Canad. Conf. Comput. Geom., pages 269-274, 1994.

[5] J. Fiala, J. Kratochvil, and A. Proskurowski. Systems of distant representatives. Discrete Applied Mathematics, 145:306-316, 2005.

[6] L. J. Guibas, D. Salesin, and J. Stolfi. Constructing strongly convex approximate hulls with inaccurate primitives. Algorithmica, 9:534-560, 1993.

[7] S. Jadhav, A. Mukhopadhyay, and B. K. Bhattacharya. An optimal algorithm for the intersection radius of a set of convex polygons. J. Algorithms, 20:244-267, 1996.

[8] M. Löffler and M. van Kreveld. Largest and smallest convex hulls for imprecise points. Technical Report UU-CS-2006-019, Utrecht University, Institute of Information and Computing Sciences, May 2006.

[9] M. Löffler and M. van Kreveld. Largest and smallest tours and convex hulls for imprecise points. In Proc. 10th Scandinavian Workshop on Algorithm Theory, LNCS 4059, pages 375$387,2006$.

[10] N. Megiddo. Linear-time algorithms for linear programming in $R^{3}$ and related problems. SIAM J. Comput., 12(4):759-776, 1983.

[11] N. Megiddo. On the ball spanned by balls. Discrete Comput. Geom., 4:605-610, 1989.

[12] T. Nagai and N. Tokura. Tight error bounds of geometric problems on convex objects with imprecise coordinates. In Proc. Jap. Conf. on Discrete and Comput. Geom., pages 252-263, 2000 .

[13] T. Nagai, S. Yasutome, and N. Tokura. Convex hull problem with imprecise input and its solution. Systems and Computers in Japan, 30(3):31-42, 1999.

[14] D. Rappaport. A convex hull algorithm for discs, and applications. Comput. Geom. Theory Appl., 1(3):171-181, 1992.

[15] D. Rappaport. Minimum polygon transversals of line segments. Internat. J. Comput. Geom. Appl., 5:243-256, 1995.

[16] J. Robert and G. Toussaint. Computational geometry and facility location. Technical Report SOCS 90.20, McGill Univ., Montreal, PQ, 1990.

[17] M. I. Shamos. Computational Geometry. Ph.D. thesis, Dept. Comput. Sci., Yale Univ., New Haven, CT, 1978.

[18] E. Welzl. Smallest enclosing discs (balls and ellipsoids). In H. Maurer, editor, New Results and New Trends in Computer Science, LNCS 555, pages 359-370. Springer Verlag, 1991.

[19] C. K. Yap. An $O(n \log n)$ algorithm for the Voronoi diagram of a set of simple curve segments. Discrete Comput. Geom., 2:365-393, 1987. 